International Journal of Pure and Applied Mathematics

Volume 90 No. $1 \quad 2014,5-9$

ISSN: 1311-8080 (printed version); ISSN: 1314-3395 (on-line version)

url: http://www.ijpam.eu

doi: http://dx.doi.org/10.12732/ijpam.v90i1.2

ijpam.eu

\title{
A NON-UNIFORM BOUND ON POISSON APPROXIMATION FOR RANDOM SUMS OF GEOMETRIC RANDOM VARIABLES
}

\author{
K. Teerapabolarn \\ Department of Mathematics \\ Faculty of Science \\ Burapha University \\ Chonburi, 20131, THAILAND
}

\begin{abstract}
We determine a non-uniform bound for the distance between the distribution function of random sums of independent geometric random variables and an appropriate Poisson distribution function. Two examples have been given to illustrate the result obtained.
\end{abstract}

AMS Subject Classification: 62E17, 60F05, 60G05

Key Words: distribution function, geometric random variable, Poisson approximation, random sums, Stein-Chen method

\section{Introduction}

Let $S_{n}$ be a sum $\sum_{i=1}^{n} X_{i}$ of independently distributed geometric random variables, where $P\left(X_{i}=k\right)=\left(1-p_{i}\right)^{k} p_{i}$ for $k=0,1, \ldots$ It is well-known that if all $q_{i}=\left(1-p_{i}\right)$ are small, the distribution of $S_{n}$ can be approximated by the Poisson distribution with mean $E\left(S_{n}\right)=\sum_{i=1}^{n} q_{i} p_{i}^{-1}$. Correspondingly, the distribution function of $S_{n}$ can also be approximated by Poisson distribution function with these means. Let $\mathbb{P S}_{n}\left(x_{0}\right)=P\left(S_{n} \leq x_{0}\right)$ and $\mathbb{P}_{\lambda_{n}}\left(x_{0}\right)=\sum_{k=0}^{x_{0}} \frac{e^{-\lambda_{n}} \lambda_{n}^{k}}{k !}$ be the distribution function of $S_{n}$ and the Poisson distribution function with mean $\lambda_{n}$

Received: July 17, 2013

(c) 2014 Academic Publications, Ltd. url: www.acadpubl.eu 
at $x_{0} \in \mathbb{N}$, respectively. In this case, Teerapabolarn [4] used the Stein-Chen method to give a non-uniform bound for the difference of $\mathbb{P S}_{n}\left(x_{0}\right)$ and $\mathbb{P}_{\lambda_{n}}\left(x_{0}\right)$ as follows:

$$
\left|\mathbb{P S}_{n}\left(x_{0}\right)-\mathbb{P}_{\lambda_{n}}\left(x_{0}\right)\right| \leq \min \left\{\frac{1-e^{-\lambda_{n}}}{\lambda_{n}}, \frac{1}{x_{0}}\right\} \sum_{i=1}^{n} q_{i}^{2} p_{i}^{-2},
$$

where $x_{0} \in \mathbb{N}$. Consider the sum $S_{N}=\sum_{i=1}^{N} X_{i}$, where $N$ is a non-negative integer valued random variable and independent of the $X_{i}$ 's. Then $S_{N}$ is called the random sums of independent geometric random random variables. In this study, we are interest to approximate $\mathbb{P S}_{N}\left(x_{0}\right)$ by $\mathbb{P}_{\lambda}\left(x_{0}\right)$ when $\lambda=E\left(\lambda_{N}\right)$.

\section{Method}

Stein's method was originally formulated for normal approximation by Stein [2]. It was adapted and applied to the Poisson case by Chen [1], which is refer to as the Stein-Chen method. Following [3], Stein's equation of the Poisson cumulative distribution function with parameter $\lambda>0$ is of the form

$$
h_{x_{0}}(x)-\mathbb{P}_{\lambda}\left(x_{0}\right)=\lambda f_{x_{0}}(x+1)-x f_{x_{0}}(x),
$$

where $x_{0}, x \in \mathbb{N} \cup\{0\}$, and for $h_{x_{0}}(x)=1$ if $x \leq x_{0}$ and $h_{x_{0}}(x)=0$ if $x>x_{0}$, the solution $f_{x_{0}}$ is

$$
f_{x_{0}}(x)= \begin{cases}(x-1) ! \lambda^{-x} e^{\lambda}\left[\mathbb{P}_{\lambda}(x-1)\left[1-\mathbb{P}_{\lambda}\left(x_{0}\right)\right]\right], & \text { if } x \leq x_{0} \\ (x-1) ! \lambda^{-x} e^{\lambda}\left[\mathbb{P}_{\lambda}\left(x_{0}\right)\left[1-\mathbb{P}_{\lambda}(x-1)\right]\right], & \text { if } x>x_{0} \\ 0, & \text { if } x=0\end{cases}
$$

The following lemma gives a non-uniform bound of (2.2).

Lemma 2.1. For $x_{0} \in \mathbb{N}$, let $p_{\lambda}\left(x_{0}+1\right)=\frac{e^{-\lambda} \lambda_{0}+1}{\left(x_{0}+1\right) !}$. Then the following inequality holds:

$$
0 \leq \sup _{x \geq 1} f_{x_{0}}(x) \leq \frac{\mathbb{P}_{\lambda}\left(x_{0}\right)\left(1-\mathbb{P}_{\lambda}\left(x_{0}\right)\right)}{\left(x_{0}+1\right) p_{\lambda}\left(x_{0}+1\right)}[3]
$$




\section{Result}

The following theorem presents non-uniform bounds with different Poisson mean for the distance between $\mathbb{P S}_{N}\left(x_{0}\right)$ and $\mathbb{P}_{\lambda}\left(x_{0}\right)$.

Theorem 3.1. Let $\lambda=E\left(\lambda_{N}\right)$ and $x_{0} \in \mathbb{N}$, then we have

$$
\begin{aligned}
\left|\mathbb{P S}_{N}\left(x_{0}\right)-\mathbb{P}_{\lambda}\left(x_{0}\right)\right| \leq \min & \left\{E\left(\frac{1-e^{-\lambda_{N}}}{\lambda_{N}} \sum_{i=1}^{N} q_{i}^{2} p_{i}^{-2}\right), \frac{E\left(\sum_{i=1}^{N} q_{i}^{2} p_{i}^{-2}\right)}{x_{0}}\right\} \\
& +\frac{\mathbb{P}_{\lambda}\left(x_{0}\right)\left(1-\mathbb{P}_{\lambda}\left(x_{0}\right)\right) E\left|\lambda_{N}-\lambda\right|}{\left(x_{0}+1\right) p_{\lambda}\left(x_{0}+1\right)}
\end{aligned}
$$

where $\mathbb{P S}_{N}(0)=E\left(\prod_{i=1}^{N} p_{i}\right)$.

Proof. 1. We have

$$
\begin{aligned}
\left|\mathbb{P S}_{N}\left(x_{0}\right)-\mathbb{P}_{\lambda}\left(x_{0}\right)\right| \leq & \left|\mathbb{P S}_{N}\left(x_{0}\right)-\mathbb{P}_{\lambda_{N}}\left(x_{0}\right)\right|+\left|\mathbb{P}_{\lambda_{N}}\left(x_{0}\right)-\mathbb{P}_{\lambda}\left(x_{0}\right)\right| \\
= & \sum_{n=0}^{\infty} P(N=n)\left|\mathbb{P S}_{n}\left(x_{0}\right)-\mathbb{P}_{\lambda_{n}}\left(x_{0}\right)\right|+\left|\mathbb{P}_{\lambda_{N}}\left(x_{0}\right)-\mathbb{P}_{\lambda}\left(x_{0}\right)\right| \\
\leq & \sum_{n=0}^{\infty} P(N=n) \min \left\{\frac{1-e^{-\lambda_{n}}}{\lambda_{n}}, \frac{1}{x_{0}}\right\} \sum_{i=1}^{n} q_{i}^{2} p_{i}^{-2} \\
& \quad+\left|\mathbb{P}_{\lambda_{N}}\left(x_{0}\right)-\mathbb{P}_{\lambda}\left(x_{0}\right)\right|,
\end{aligned}
$$

where the first term of the right hand side of (3.2) follows from (1.1). Because

$$
\begin{aligned}
\left|\mathbb{P}_{\lambda_{N}}\left(x_{0}\right)-\mathbb{P}_{\lambda}\left(x_{0}\right)\right| & =\left|E\left\{\lambda f_{x_{0}}\left(U_{\lambda_{N}}+1\right)-U_{\lambda_{N}} f_{x_{0}}\left(U_{\lambda_{N}}\right)\right\}\right| \\
& =\left|E\left\{\lambda E\left\{\left[f_{x_{0}}\left(U_{\lambda_{N}}+1\right)\right] \mid \lambda_{N}\right\}-E\left\{\left[U_{\lambda_{N}} f_{x_{0}}\left(U_{\lambda_{N}}\right)\right] \mid \lambda_{N}\right\}\right\}\right| \\
& =\left|E\left\{\lambda E\left\{\left[f_{x_{0}}\left(U_{\lambda_{N}}+1\right)\right] \mid \lambda_{N}\right\}-\lambda_{N} E\left\{\left[f_{x_{0}}\left(U_{\lambda_{N}}+1\right)\right] \mid \lambda_{N}\right\}\right\}\right| \\
& =\left|E\left\{\left(\lambda-\lambda_{N}\right) E\left[f_{x_{0}}\left(U_{\lambda_{N}}+1\right) \mid \lambda_{N}\right]\right\}\right| \\
& \leq \sup _{x \geq 1} f_{x_{0}}(x) E\left|\lambda_{N}-\lambda\right| \\
& \leq \frac{\mathbb{P}_{\lambda}\left(x_{0}\right)\left(1-\mathbb{P}_{\lambda}\left(x_{0}\right)\right) E\left|\lambda_{N}-\lambda\right|}{\left(x_{0}+1\right) p_{\lambda}\left(x_{0}+1\right)}
\end{aligned}
$$

Combining (3.2) and (3.3), the proof is complete.

If $X_{i}$ 's are identically distributed, then the following corollary is an immediately consequence of the Theorem 3.1 
Corollary 3.1. If $p_{1}=p_{2}=\cdots=p$, then we have the following:

$$
\begin{aligned}
\left|\mathbb{P S}_{N}\left(x_{0}\right)-\mathbb{P}_{\lambda}\left(x_{0}\right)\right| \leq \min & \left\{E\left(1-e^{-\lambda_{N}}\right) q p^{-1}, \frac{E(N) q^{2} p^{-2}}{x_{0}}\right\} \\
+ & \frac{\mathbb{P}_{\lambda}\left(x_{0}\right)\left(1-\mathbb{P}_{\lambda}\left(x_{0}\right)\right) E|N-E(N)| q p^{-1}}{\left(x_{0}+1\right) p_{\lambda}\left(x_{0}+1\right)} .
\end{aligned}
$$

\section{Examples}

We give two examples to illustrate the result in the case of $X_{i}$ 's are identically distributed.

Example 4.1. For $n(n \in \mathbb{N})$ is fixed, let $N$ be a positive integer-valued random variable with probability function

$$
P(N=k)= \begin{cases}\frac{1}{2}, & k=n \\ \frac{1}{2}, & k=2 n \\ 0, & \text { otherwise }\end{cases}
$$

Therefore $E(N)=\frac{3 n}{2}$ and $E|N-E(N)|=\frac{n}{2}$. Let $p_{1}=p_{2}=\cdots=p$, then for $x_{0} \in \mathbb{N}$, we have

$$
\left|\mathbb{P S}_{N}\left(x_{0}\right)-\mathbb{P}_{\lambda}\left(x_{0}\right)\right| \leq \min \left\{q p^{-1}, \frac{3 n q^{2} p^{-2}}{2 x_{0}}\right\}+\frac{\mathbb{P}_{\lambda}\left(x_{0}\right)\left(1-\mathbb{P}_{\lambda}\left(x_{0}\right)\right) n q p^{-1}}{2\left(x_{0}+1\right) p_{\lambda}\left(x_{0}+1\right)}
$$

Example 4.2. Let $N$ be a positive integer-valued random variable with probability function

$$
P(N=n)=\frac{1}{2^{n}}, n=1,2, \ldots,
$$

then we have $E(N)=2$ and $E|N-E(N)|=1$. If $p_{1}=p_{2}=\cdots=p$, then for $x_{0} \in \mathbb{N}$, we obtain

$$
\left|\mathbb{P S}_{N}\left(x_{0}\right)-\mathbb{P}_{\lambda}\left(x_{0}\right)\right| \leq \min \left\{q p^{-1}, \frac{2 q^{2} p^{-2}}{x_{0}}\right\}+\frac{\mathbb{P}_{\lambda}\left(x_{0}\right)\left(1-\mathbb{P}_{\lambda}\left(x_{0}\right)\right) q p^{-1}}{\left(x_{0}+1\right) p_{\lambda}\left(x_{0}+1\right)}
$$




\section{References}

[1] L.H.Y. Chen, Poisson approximation for dependent trials, Ann. Probab., 3 (1975), 534-545.

[2] C.M. Stein, A bound for the error in normal approximation to the distribution of a sum of dependent random variables, Proc. Sixth Berkeley Sympos. Math. Statist. Probab., 3 (1972), 583-602.

[3] K. Teerapabolarn, A new bound on Poisson approximation for independent geometric variables, Int. J. Pure Appl. Math., 84 (2013), 419-422.

[4] K. Teerapabolarn, Improvement of Poisson approximation for independent geometric trials, Int. J. Pure Appl. Math., 87 (2013), 273-276. 
